\title{
Effectiveness of using a meditation app in reducing anxiety and improving well-being during the COVID-19 pandemic: A structured summary of a study protocol for a randomized controlled trial
}

\author{
Katherine T. O'Donnell, Melanie Dunbar and Diana L. Speelman *i)
}

\begin{abstract}
Objectives: This interventional study will investigate the effect of daily use of a mindfulness app on measures of participant anxiety, well-being, and perceived outlook during the COVID-19 pandemic, by comparing preintervention survey responses to post-intervention survey responses.

Trial design: Randomized, controlled trial with parallel assignment. Adults will be assigned either to daily use of a meditation app for 30 days or to a control group (no usage of meditation app) with a 1:1 equivalence allocation ratio.

Participants: Inclusion Criteria: Participants must be 18 or older, have a smartphone, able to download apps to their smartphone, must be fluent in the English language, able to complete surveys on their own, and must be in the United States for the duration of the study. Exclusion Criteria: Current regular use of a mindfulness or meditation app, regular practice of mindfulness or meditation, regular therapy sessions, inability to complete surveys independently, or any mental health restrictions that would prevent participation. All data will be collected through the Insight Timer Meditation App and Google Forms. This trial is being conducted through the Lake Erie College of Osteopathic Medicine in Erie, PA, with all data collected digitally.
\end{abstract}

Intervention and comparator: Intervention: Participants will be sent a link to a pre-intervention survey prior to first use of the mindfulness app. Participants will be instructed to use the Insight Timer app for 10 minutes daily for 30 days. At the end of the 30-day intervention period, participants will be sent a link for the post-intervention survey. Two months after the conclusion of the 30-day intervention period, participants will be sent a link for another post-intervention survey. Comparator: Participants will receive the same surveys, but will not use any mindfulness app for the 30-day intervention period. After this 30-day period, participants are invited to use the Insight Timer app if they so choose.

* Correspondence: dspeelman@lecom.edu

Lake Erie College of Osteopathic Medicine, Erie, PA, USA 
(Continued from previous page)

Main outcomes: The main outcomes are (1) anxiety as assessed by survey questions adapted from the GAD7, comparing pre-intervention to post-30-days of app usage and (2) well-being as assessed by survey questions adapted from the WHO-5, comparing pre-intervention and post-30-days of app usage.

Randomization: Participants will be allocated to interventions via a block random sequence generator with a 1:1 allocation ratio in blocks of 8.

Blinding (masking): No masking is being used in this study (open label).

Numbers to be randomized (sample size): Approximately 75 participants will be randomized to each group, with an estimated enrollment of 150 participants.

Trial status: This study is protocol version number 27-126 and was approved on May 10, 2020. Recruitment began on August 19, 2020 and will end February 28, 2021. The study is estimated to complete on April 30, 2021.

Trial registration: This trial was registered to ClinicalTrials.gov on 30 April 2020. The ClinicalTrials.gov Identifier is NCT04369378.

Full protocol: The full protocol is attached as an additional file, accessible from the Trials website (Additional File 1). In the interest in expediting dissemination of this material, the familiar formatting has been eliminated; this Letter serves as a summary of the key elements of the full protocol.

Keywords: COVID-19, Randomized controlled trial, protocol, mindfulness, well-being, anxiety, meditation app

\section{Supplementary Information}

The online version contains supplementary material available at https://doi. org/10.1186/s13063-020-04935-6.

Additional file 1. Full Study Protocol.

\section{Acknowledgements}

Not applicable.

\section{Authors' contributions}

Conceptualization: $\mathrm{KO}$; methodology: KO, MD, DS; human subjects ethics protocol: $\mathrm{KO}, \mathrm{MD}$, DS; data collection: $\mathrm{KO}$, DS; data analysis: $\mathrm{KO}$, DS; writing of manuscript: DS; manuscript review and editing: KO, MD, DS. All authors read and approved the final manuscript.

\section{Funding}

There are no sources of funding for this study.

\section{Availability of data and materials}

De-identified participant data will be reported as individual data points, as well as means +/- SD or medians with quartiles upon publication of findings. Access criteria to the final trial dataset will be upon written request to the corresponding author on publication.

\section{Ethics approval and consent to participate}

This trial under the protocol reference number 27-126 received ethical approval from the Institutional Review Board at Lake Erie College of Osteopathic Medicine on May 10, 2020, with addendums approved on June 30, 2020 and August 17, 2020. Investigators certify that this trial has received ethical approval from the appropriate ethical committee as described above. Intentions to obtain consent from participants to participate in this study include a Google Form Enrollment Survey listing protocol for procedures, confidentiality, risks, benefits, voluntary participation, right to withdraw, new findings, research resources, and mental health resources. Participants will then again read eligibility criteria and respond to the survey, agreeing that they are in fact eligible to participate and that they have read the study information and consent statement, and that they consent to participate in this study. The survey also asks participants to provide an email address to be used to receive a participant ID number, surveys, and instructions for downloading and installing the mindfulness app.

\section{Consent for publication}

Not applicable.

\section{Competing interests}

The authors declare that they have no competing interests.

Received: 19 November 2020 Accepted: 25 November 2020

Published online: 09 December 2020

\section{Publisher's Note}

Springer Nature remains neutral with regard to jurisdictional claims in published maps and institutional affiliations.

Ready to submit your research? Choose BMC and benefit from:

- fast, convenient online submission

- thorough peer review by experienced researchers in your field

- rapid publication on acceptance

- support for research data, including large and complex data types

- gold Open Access which fosters wider collaboration and increased citations

- maximum visibility for your research: over $100 \mathrm{M}$ website views per year

At BMC, research is always in progress.

Learn more biomedcentral.com/submissions 